\title{
Advancing resilience measurement
}

To the Editor - Resilience has rapidly risen to the top of the agenda for sustainability and development ${ }^{1}$. The concept underpins a number of high-level policy initiatives, including the Sustainable Development Goals and Paris Agreement. In the face of the ongoing COVID-19 pandemic, and with billions of dollars' worth of resilience investments being mobilized globally, resilience will continue to rise as a policy priority. The scale of these investments further amplifies the importance of resilience and highlights the need for rigorous and decision-oriented evidence. Under these conditions, we believe that current measurement approaches can do more to serve the learning, evaluation and accountability needs of resilience stakeholders.

Efforts to measure resilience face conceptual and methodological hurdles ${ }^{2}$. Resilience has differing entry points, definitions and applications across disciplines. Many sustainability scholars and practitioners draw on frameworks that have roots in socio-ecological systems $(\mathrm{SES})^{3}$. These typically share a commitment to understanding how and why individuals and/or systems cope, adapt and potentially transform in response to disturbance ${ }^{4}$. While research on SES has infused new perspectives into development practice, including systems thinking and complexity ${ }^{5}$, conceptual diversity continues to limit efforts at standardizing metrics. As a result, the number of resilience measurement approaches has risen rapidly, with many stakeholders struggling to match their evidence needs with suitable tools ${ }^{6}$.

Insights from a growing body of research and a range of development and humanitarian initiatives ${ }^{7}$ further underline the need for greater innovation and methodological rigour in the resilience evidence base. It is against this backdrop that we propose a forward-looking agenda for resilience measurement, summarized in Box 1 - one that can foster robust, verifiable and credible evidence on what works (and what doesn't) for resilience-building efforts.

\section{An action agenda}

While the diversity of resilience measurement approaches provides an opportunity for different perspectives to emerge, it makes comparisons difficult. Key to developing a robust body of resilience evidence - that is, one that demonstrates a commitment to precision, accuracy and

\section{Box 1 | Priorities for the next generation of resilience measurement}

This agenda is informed by priorities expressed by leaders from United Nations agencies, policy makers from national governments, and measurement experts at a high-level consultation on resilience evidence held in Dakar, Senegal in December 2018.

Priority 1. Promote shared principles for robust and harmonized measurement. Use of guiding principles can help to harmonize the resilience evidence base, promoting methodological rigour and ease of comparability across frameworks.

Priority 2. Leverage technological innovations and new data opportunities. Greater use of remote data collection,

verifiability - is establishing common guiding measurement principles. Early work by the Resilience Measurement Technical Working Group has begun to lay the foundations ${ }^{8}$. Building on a wide range of practical applications, we support their call to: (1) better understand the varied impacts of shock events; (2) place well-being as a core measurement outcome; (3) identify capacities that maintain well-being in the face of shocks; and (4) draw on contextual factors to explain regional variation in resilience. Putting these guidelines into practice is key to matching funder and practitioner needs with the right measurement tools, as well as building stakeholder trust in the resilience evidence base.

Measuring progress in achieving outcomes is inherently data intensive. It requires complex datasets and indicators from varying scales across multiple sectors. It is here that technological innovations can play an important role. One way is to promote greater use of remote data collection and Earth observations in tracking resilience outcomes. For example, in Myanmar, mobile phone panel surveys provide real-time updates (both quantitative and qualitative) on levels of household flood resilience without the need for on-the-ground survey teams ${ }^{9}$. Similarly, large-scale efforts to use phones in tracking the impacts of COVID-19 globally are a reassuring sign ${ }^{10}$. These tools offer promise in addressing many of the timing and access challenges that currently hinder resilience
Earth observations and big data analytics can unlock new insights into resilience dynamics, particularly in post-disaster contexts.

Priority 3. Encourage rigorous impact assessments. Investments in counterfactual mixed-methods impact assessments are vital to warrant claims of impact and to understand causal mechanisms for resilience building.

Priority 4. Generate resilience evidence to inform policy. Collaboration among policy makers, implementers and analysts in support of resilience measurement helps to ensure that evidence speaks directly to diverse user needs.

measurement, particularly in fragile and conflict-affected areas. Research also hints at opportunities provided by machine learning to identify better predictors of resilience and highlight hotspots of vulnerability using neural networks ${ }^{11,12}$. Resilience measurement must harness these analytical tools, feeding decision makers with novel insights on people's dynamic ability to deal with risk.

Establishing causality is crucial to determining intended (and unintended) impacts of interventions. Impact assessments with careful causal designs infer whether gains can be attributed to a given resilience intervention or other external factors that influence well-being. Many impact assessments still fail to employ measurement approaches that support causal inference. Resilience is no exception: the number of causal studies pales in comparison with wider research efforts. The next generation of resilience measurement needs to invest more in counterfactual approaches, including randomization, instrumental variables and regression discontinuity designs ${ }^{13}$. Alongside this, qualitative methods must play a vital role in helping to identify and explain causal mechanisms, especially in light of the contextualized nature of resilience ${ }^{14}$. Development funders should earmark support for mixed-methods impact assessment to better understand constraints and opportunities in building resilience.

A lack of synergy among resilience stakeholders is far too common. There are three ways to ensure resilience 
measurement can better serve diverse user needs. First, resilience measurement must have the capacity to be rapidly deployed at critical junctures - requiring practical, less-time-intensive measurement tools. Second, evidence needs will evolve during different stages of risk preparedness and recovery: while quick measurement snapshots may be sufficient post-shock, longer-term assessments are needed where communities are focused on sustained development gains. Third, resilience measurement should be organized as a collaborative effort, whereby policy makers, implementers and analysts work together in designing the measurement process. For example, embedding of early-career researchers into local municipalities has enhanced co-produced knowledge of resilience in a number of southern African cities ${ }^{15}$. These collaborations ensure that measurement tools are better refined and contextualized to deepen our understanding of how resilience is built.
Bringing the proposed action agenda to life necessitates concerted action by all those committed to improving the quality of resilience measurement. As resilience is increasingly featured in large-scale sustainable development investments, we hope that the priorities highlighted here provide a catalyst for action to advance a more impactful measurement agenda.

Lindsey Jones (DD $1 \times$, Mark A. Constas ${ }^{2}$, Nathanial Matthews ${ }^{(D)}{ }^{3}$ and Simone Verkaart (D) 3

${ }^{1}$ London School of Economics and Political Science, London, UK. ${ }^{2}$ Cornell University, Ithaca, NY, USA. ${ }^{3}$ Global Resilience Partnership, Stockholm, Sweden. 凶e-mail: l.jones3@lse.ac.uk

Published online: 4 January 2021

https://doi.org/10.1038/s41893-020-00642-x

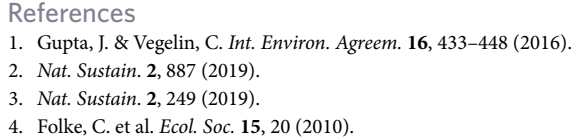

5. Allen, C. R., Angeler, D. G., Chaffin, B. C., Twidwell, D. \& Garmestani, A. Nat. Sustain. 2, 898-900 (2019).

6. Grafton, R. Q. et al. Nat. Sustain. 2, 907-913 (2019).

7. Resilience Insights: Lessons from the Global Resilience Partnership (Global Resilience Partnership, 2019).

8. Constas, M. A. et al. A Common Analytical Model for Resilience Measurement: A Causal Framework and Some Methodological Options (Food Security Information Network, 2014).

9. Jones, L. \& Ballon, P. Glob. Environ. Change 62, 102053 (2020) 10. High Frequency Mobile Phone Surveys of Households to Assess the Impacts of COVID-19 (World Bank 2020).

11. Knippenberg, E., Jensen, N. \& Constas, M. World Dev. 121, 1-15 (2019).

12. Machine Learning for Disaster Risk Management (Global Facility for Disaster Risk Reduction, 2018).

13. Gertler, P., Martinez, S., Premand, P., Rawlings, L. \& Vermeersch, C. Impact Evaluation in Practice (World Bank, 2011).

14. Maxwell, D., Constas, M., Frankenberger, T., Klaus, D. \& Mock, N. Qualitative Data and Subjective Indicators for Resilience Measurement (Food Security Information Network, 2015).

15. Embedded Researchers Bridge Research and Decision-Making to Build the Climate Resilience of Southern African Cities (Future Resilience for African Cities and Lands, 2019).

Competing interests

The authors declare no competing interests.

Additional information

Peer review information Nature Sustainability thanks the anonymous reviewers for their contribution to the peer review of this work. 